\title{
State-of-practice on As-Is Modelling of Industrial Facilities
}

\author{
Eva Agapaki ${ }^{10000-0002-2962-9203]}$ and Ioannis Brilakis ${ }^{2[0000-0003-1829-2083]}$ \\ ${ }^{1}$ Ph.D. Candidate, Department of Engineering, University of Cambridge, CB2 1PZ, U.K. \\ ea 437 @cam. ac.uk \\ ${ }^{2}$ Laing O'Rourke Reader, Department of Engineering, University of Cambridge, CB2 1PZ, \\ U.K. \\ ib340@cam.ac.uk
}

\begin{abstract}
D models of industrial facilities is spent on geometric modelling due to the sheer number of Industrial Objects (IOs) of each plant. Hence, cost reduction is only possible by automating modelling. Our previous work has successfully identified the most frequent industrial objects which are in descending order: electrical conduit, straight pipes, circular hollow sections, elbows, channels, solid bars, I-beams, angles, flanges and valves. We modelled those on a state-of-theart software, EdgeWise and then evaluated the performance of this software for pipeline and structural modelling. The modelling of pipelines is summarized in three basic steps: (a) automated extraction of cylinders, (b) their semantic classification and (c) manual extraction and editing of pipes. The results showed that cylinders are modelled with $75 \%$ recall and $62 \%$ precision on average. We discovered that pipes, electrical conduit and circular hollow sections require 80 $\%$ of the Total Modelling Hours (TMH) of the 10 most frequent IOs to build the plant model. TMH was then compared to modelling hours in Revit and showed that $67 \%$ of pipe modelling time is saved by EdgeWise. This paper is the first to evaluate state-of-the-art industrial modelling software. These findings help in better understanding the problem and serve as the foundation for researchers who are interested in solving it.
\end{abstract}

Keywords: Industrial Facilities, Facility Management, Building Information Modelling.

\section{Introduction}

"As-Is" Building Information Models (AI-BIMs) are the 3D digital representation of the existing condition of facilities and encompass geometric definitions at different levels of aggregation and parametric rules [1]. The clear majority of large refineries were built before the advent of CAD in 1977: as-is models, therefore, do not exist to assist their maintenance operations [2, 3]. AI-BIMs of industrial plants have substantial impact in various applications. Some of these include maintenance, strategic plan- 
ning of their operations, revamping purposes, retrofitting of old sites and preparation for dismantling [4-7].

Inexistence of AI-BIMs will result in time lags for these operations. This is crucial for industrial managers, since without detailed planning, productivity will be substantially affected, and the agreed budget and timeline expectations will not be met. Moreover, there are thresholds on the acceptable shut down duration that will not impede production, and those limits cannot be violated without incurring extra costs. For instance, [8] reported that $40 \%$ of the total 3D modelling cost of retrofitting a Chevron plant was spent on data-processing labor and the shut-down time was limited to 72 hours to avoid additional costs. Every modelling hour saved can prevent critical failures or unexpected accidents, thus continuous production flow of these assets is achieved. This work aims to assist the tedious current practice in this regard.

Modelers use the following four main steps to manually process AI-BIMs: (a) data collection, (b) point cloud registration, (c) geometric modelling and (d) addition of accompanying information. Initially, data is collected using laser scanners and photogrammetry, which are represented by their cartesian or polar coordinates, the point cloud, and in some cases by their color data (RGB). The scans need to be registered in a consistent coordinate system by calculating inter-scan rigid body transformations and the registered point cloud represents the complete measured data. Then this data needs to be geometrically modelled.

Geometric modelling entails (a) primitive shape detection, (b) semantic classification of detected shapes and (c) fitting. Firstly, primitive shapes are detected (e.g., cylinders, tori, planes) and labelled (e.g., pipes, elbows, I-beams). Afterwards, the primitives are fitted to known solid shapes to obtain their geometric parameters. Their relationships to other objects need to be obtained in order to produce a complete AIBIM in the Industry Foundation Schema (IFC) format. IFC is a data format that allows geometric, material and other construction related information to coexist in a single model.

Geometric modelling is the "bottleneck" during the Scan-to-BIM process of any industrial facility given how costly and time consuming it is. Recent studies have reported that geometric processing takes $90 \%$ of the modelling time $[9,10]$. [10] reported that 10 operators were needed to process 1084 scans of a nuclear reactor and model its objects in around 6 months using Dassault Systems SolidWorks and Trimble Realworks. In contrast, laser scanning of the plant was completed in only 35 days. This significant time required to model the vast number of industrial objects impedes adoption of as-is 3D modelling for these plants.

The research presented in this paper is exploratory in nature, not causal. It does not seek to solve the problem of automating the modelling of industrial facilities. It rather seeks to improve our understanding of the problem and the extent to which it has been resolved so far and provide a foundation for future researchers interested in solving it. This is why the main objective of this paper is to identify how laborious industrial objects are for modelling, as well as to measure the performance of existing tools in modelling these particular object types. The authors used the most frequent objects based on a statistical analysis of 3D modelled industrial objects in a variety of industrial plants as explained in [11]. An overview of the state-of-the-art tools available for 
as-is modelling is given to select the most advanced tool for evaluation. The most frequent objects were modelled in the state-of-the-art, semi-automated modelling software, EdgeWise, and their average modelling time was measured. The level of automation of EdgeWise is also measured for the most frequent industrial object types. This analysis will substantially assist automated modelling efforts to efficiently reduce modelling time and facilitate facility management.

\section{Background}

Industrial plants can be divided into ten main categories [12]: (a) onshore and (b) offshore oil platforms, (c) chemical, (d) mining, (e) pharmaceutical plants, (f) power plants, $(\mathrm{g})$ water and wastewater treatment facilities, (h) natural gas processing and biochemical plants, (i) refineries, (j) food processing factories, (k) defense facilities, (l) metal production facilities, (m) nuclear plants, (n) research facilities and (o) warehouses and silos. The object types of industrial facilities belong to the main object categories: (a) structural elements, (b) piping system, (c) electrical, (d) safety and (e) general equipment, (f) architectural elements, (g) instrumentation, (h) Heating, Ventilation and Air Conditioning (HVAC) and (i) civil elements.

The most frequent object categories being around $90 \%$ of all objects in these facilities are: structural elements (33\%), the piping system (28\%) and electrical equipment (27\%) based on our previous work [11]. The most frequent object types of these categories are in descending order: electrical conduit (24\%), straight pipes $(15 \%)$, circular hollow sections $(6.5 \%)$, elbows $(5.4 \%)$, channels $(5 \%)$, solid bars $(4.5 \%)$, I-beams (4.4\%), angles ( $4 \%$ ), flanges $(3.3 \%)$ and valves $(2 \%)$ as presented in the same work.

\subsection{Automated industrial plant modelling}

\section{State-of-the-art software}

Almost all available modelling tools of industrial objects depend on human intervention for most of the modelling tasks. Leading 3D CAD software (Autodesk, Bentley, AVEVA and FARO) have developed programs containing a variety of functions that enable 3D plant modelling and visualization from 3D point clouds. For example, AutoCAD Plant 3D accompanied with FARO's PointSense Plant add-in enables semi-automated pipe modelling from Point Clouds. PointSense Plant provides several functions and a large standard library with a variety of piping and structural components available for the detection of pipelines from 3D point clouds. Moreover, fitting template objects to scanned 3D objects is performed automatically and constraints can be applied to fix potential errors of fitting. PointSense Plant 17.5 has integrated a precalculation tool that detects cylinders in the point cloud of a specific area and has the ability to colorize the Point Cloud by deviation from reference geometry [13]. However, the users still manually model the as-is pipelines by finding the insertion points for fitting CAD objects to the segmented 3D point clouds and fitting errors of the extracted cylinders are not provided. The "Walk the Run" feature is rather a suggestion for pipe insertion points than an automated pipe modelling tool. 
EdgeWise is another semi-automated platform that is extensively used. The main difference of Pointsense and EdgeWise is that a modeler using the former should extract the desired boundaries of an object manually and afterwards the software will automatically extract the correct dimensions and location. However, this procedure is automatically performed by EdgeWise, that is why it was chosen as the most suitable tool for evaluation of the most frequent industrial object types that will be presented in the next section. Structural sections are modelled manually in all available software packages. Fitting of user-selected primitives (e.g., cylinders, cuboids, tori etc) is performed automatically by both EdgeWise and PointSense Plant. To date, no one has provided viable assessments of state-of-the-art tools.

The details behind the algorithmic development of software are commercially protected (trade secrets), so we can evaluate only the outcomes and not the algorithms that are used to reach those outcomes.

\section{State-of-research}

State-of-the-art research work on pipe detection has partially solved the problem and not to a greater extent compared to commercially available software like EdgeWise [14, 15]. For instance, [14] only detect pipes in orthogonal directions. A recent study completed by [15] is dependent on threshold values for radius and normal estimation. The pipe radius range is $0.0254 \mathrm{~m}-0.762 \mathrm{~m}$ and the normal deviation is $5^{\circ}$. Therefore, [15] cannot be generalized for pipe detection. Their updated Hough Transform based on [4] study detects pipes in two sample datasets with $60 \%$ recall and 89 $\%$ precision.

Prior knowledge of industrial scenes has assisted researchers to detect industrial objects. [16] used prior knowledge (Piping and Instrumentation Diagram, P\&ID) to detect Mechanical, Electrical and Plumbing equipment (MEP). However, as-is P\&IDs are often not available as prior knowledge in industrial plants, thus they do not reflect the modifications a plant undergoes through its life. For this reason, prior knowledge cannot rely on P\&IDs. [17] used topological information to extract semantic labels for four object classes: pipes, planes, elbows and valves. They detect cylinders with $86 \%$ precision and $92 \%$ recall. However, their semantic labels consider that all cylindrical objects are pipes, without investigating other potential object classes with the same shape.

\subsection{Gaps in knowledge and research questions}

Considering the state of practice and body of research reviewed above, existing studies for as-is modelling of industrial plants have focused on automated detection of cylindrical objects and no scientific and viable evaluation of existing state-of-the-art software tools is provided. It is therefore still unclear (1) how much time it takes to model those in state of the art software and (2) the level of automation achieved with state of the art software. 
The aim of this work is to solve the gaps in knowledge by answering the following research questions:

a) What is the time required for modelling the most frequent object types in stateof-the-art software?

b) How can state-of-the-art as-is modelling tools be assessed in terms of automated detection of objects achieved?

\section{Research Methodology Framework}

The research conducted in this paper is exploratory in nature. The most frequent object types as derived in [11] are modelled in EdgeWise to measure the modelling time of each type and the performance of the software is evaluated. The time required for manual modelling of cylindrical objects was then compared with that measured in EdgeWise. Research efforts on automated cylinder extraction are then investigated to compare the evaluation results from EdgeWise and set the ground for future research towards minimizing the modelling time of these assets.

\subsection{Data Collection and Assumptions}

Four case studies of laser scanned industrial facilities were examined to have a representative sample of industrial objects in different facilities. Two case studies are rooms of an industrial plant, one was a water treatment facility in Cambridge and the fourth was a room of a petrochemical plant. The industrial and petrochemical plant are anonymized since rights are reserved by AVEVA Group Plc. The scanner setup and scan frequency of these facilities is not available, since data was collected by industrial partners. The water treatment facility in Cambridge was laser scanned by the authors. We used a Faro Focus 3D X330 laser scanner to collect 6 laser scans with resolution of at least 1 point $/ \mathrm{cm}^{2}$ and ranging error $\pm 2 \mathrm{~mm}$.

The average frequencies of industrial objects used in this paper were taken from the results of [11] and represent the average frequency of appearance of 3D modelled datasets investigated. Solid bars are not modelled separately in EdgeWise since they cannot be distinguished from circular hollow sections in a laser survey.

\subsection{EdgeWise evaluation for pipeline modelling}

Four sample point cloud datasets were used to evaluate the capabilities of EdgeWise and obtain modelling times for the most frequent object types. Fig. 1 shows the sample datasets that were used for this evaluation.

Pipeline modelling is significantly assisted by the automated extraction of cylinders that EdgeWise provides. The scans were processed on a desktop computer with CPU Intel ${ }^{\circledR}$ Core $^{\mathrm{TM}}$ i7-4790K at $4.00 \mathrm{GHz}, 32 \mathrm{~GB}$ RAM and Windows 10 64-bit operating system. The average processing time for this operation using the abovementioned operating system for the sample datasets is $3.3 * 10^{-3} \mathrm{~min} /$ (cylinder*points in the point cloud), as shown in Table 3 . The average number of points of all datasets 
used is 258 million and the number of points of each dataset is presented in Table 1. The average diameter of cylinders and pipes is presented for evaluation purposes in Table 2.
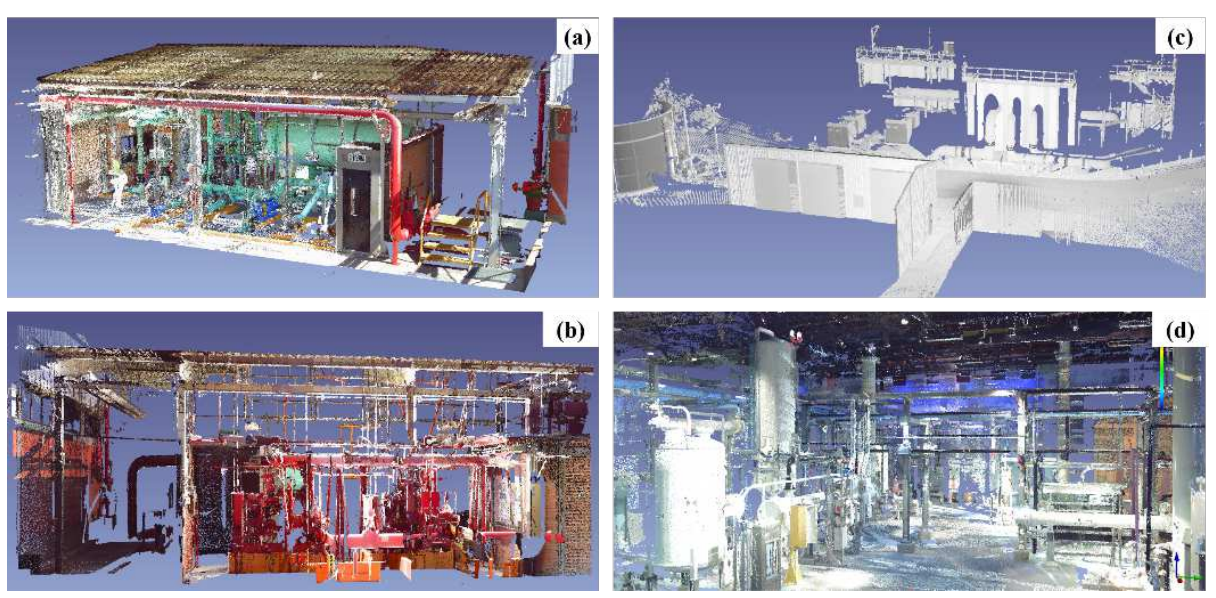

Fig. 1. Sample datasets for evaluation. (a), (b) Two rooms of a typical industrial facility, (c) a water treatment facility and (d) a room of a petrochemical plant

Table 1. Total number of points in the point cloud datasets and total number of cylinders and pipes in each case study

\begin{tabular}{lcccc}
\hline & $\begin{array}{l}\text { Typical } \\
\text { facility } \\
\text { Room 1 }\end{array}$ & $\begin{array}{l}\text { Typical } \\
\text { facility } \\
\text { Room 2 }\end{array}$ & $\begin{array}{l}\text { Water } \\
\text { Facility }\end{array}$ & $\begin{array}{l}\text { Petrochem- } \\
\text { ical plant }\end{array}$ \\
\cline { 2 - 5 } & \multicolumn{4}{c}{ Total number } \\
\hline Points (millions) & 129 & 105 & 122 & 675 \\
Automatically detected cylinders & 551 & 86 & 44 & 358 \\
Manually detected cylinders & 166 & 79 & 48 & 265 \\
\hline
\end{tabular}

We set the parameters used for cylinder extraction to a minimum of 80 points, in order to detect a pipe and provide a distance tolerance to $0.7^{*} 10^{-3} \mathrm{~m}$. The minimum threshold of the software is 50 points to identify pipelines, however if we give a very low value, the automated extraction tool will identify noisy and erroneous features as pipes. The distance tolerance is a parameter that determines how far away from the cylinder a $3 \mathrm{D}$ point can be, so that it is not excluded from the extraction algorithms. The default value of $0.7^{*} 10^{-3} \mathrm{~m}$ is used here, which was obtained from a scanner with a high level of accuracy and low noise [18].

After the automated extraction step, the cylinders were inspected and approved depending on the modeler's discretion (classification). For cases where it was difficult to identify the object, pictures taken from the laser scanner were used to assist the inspection process. A user friendly "Smart Sheet" was produced, which contains information such as the length, diameter, Root Mean Square Error (RMSE) and cover- 
age (\%) of each pipe spool. The results show that although cylinders are automatically extracted, no contextual information is provided. Henceforth, electrical conduit, handrails, cylindrical pipe supports, vessels and other object types were modelled as straight pipes.

Table 2. Average diameter of cylinders and pipes for each dataset

\begin{tabular}{lcccc}
\hline & $\begin{array}{l}\text { Typical } \\
\text { facility } \\
\text { Room 1 }\end{array}$ & $\begin{array}{l}\text { Typical } \\
\text { facility } \\
\text { Room 2 }\end{array}$ & $\begin{array}{l}\text { Water } \\
\text { Facility }\end{array}$ & $\begin{array}{l}\text { Petrochem- } \\
\text { ical plant }\end{array}$ \\
\cline { 2 - 5 } & \multicolumn{4}{c}{ Average diameter (m) } \\
\hline Cylinder & 0.067 & 0.076 & 0.315 & 0.095 \\
Pipe & 0.114 & 0.106 & 0.617 & 0.081 \\
\hline
\end{tabular}

The next step in the evaluation process was to edit the pipes and to manually add missing ones. Using the "Easy Connect" tool pipe spools were connected, and tees and elbows were added in the piping network. Then, labels were manually assigned for each cylinder that was automatically extracted by the software and metrics were used to evaluate the software's performance.

An additional step of cleaning the pipes and merging the connecting spools together was performed to complete the pipeline system. This step was completed automatically by the software. Then, standard catalogues were used to get standardized pipe dimensions. We chose the American Society of Mechanical Engineers' (ASME) specifications and pressure rating of $150 \mathrm{psi}$ for our datasets. After this step, fittings, such as flanges and valves, were applied on the standardized pipes. There are different types of standard fittings that the user can select from available standard libraries.

The modelling of pipelines is summarized in three basic steps: (a) automated extraction of cylinders, (b) semantic classification of cylinders and (c) manual extraction and editing of pipes. Fitting is performed automatically during manual or automated extraction: therefore, it is not a separate step of the procedure. The steps of the procedure for pipeline modelling in EdgeWise are presented in Fig. 2.

The average processing time per cylinder or pipe for each step is computed in Table 3 and calculated as following:

$$
\text { Time/cylinder.point }=\mathrm{TAE} / \mathrm{AC} * \mathrm{P}
$$

Where TAE is the time for automated extraction of cylinders, $\mathrm{AC}$ is the number of automatically detected cylinders and $\mathrm{P}$ is the number of points in the dataset

$$
\text { Time } / \text { pipe }=\text { TME } / \mathrm{TP}
$$

Where TME is the time for manual extraction and editing of pipes and TP is the total number of pipes

$$
\text { Time } / \text { cylinder }=\mathrm{TC} / \mathrm{AC}
$$

Where $\mathrm{TC}$ is the time for semantic classification of cylinders and $\mathrm{AC}$ is the number of automatically detected cylinders. 
The number of automatically detected cylinders and the total number of pipes is shown in Table 1 for each case study. The latter is the sum of automatically and manually detected pipes in each dataset. These normalizations are used to compare the modelling times for each case study, since the number of points and cylinders processed are different for each dataset.

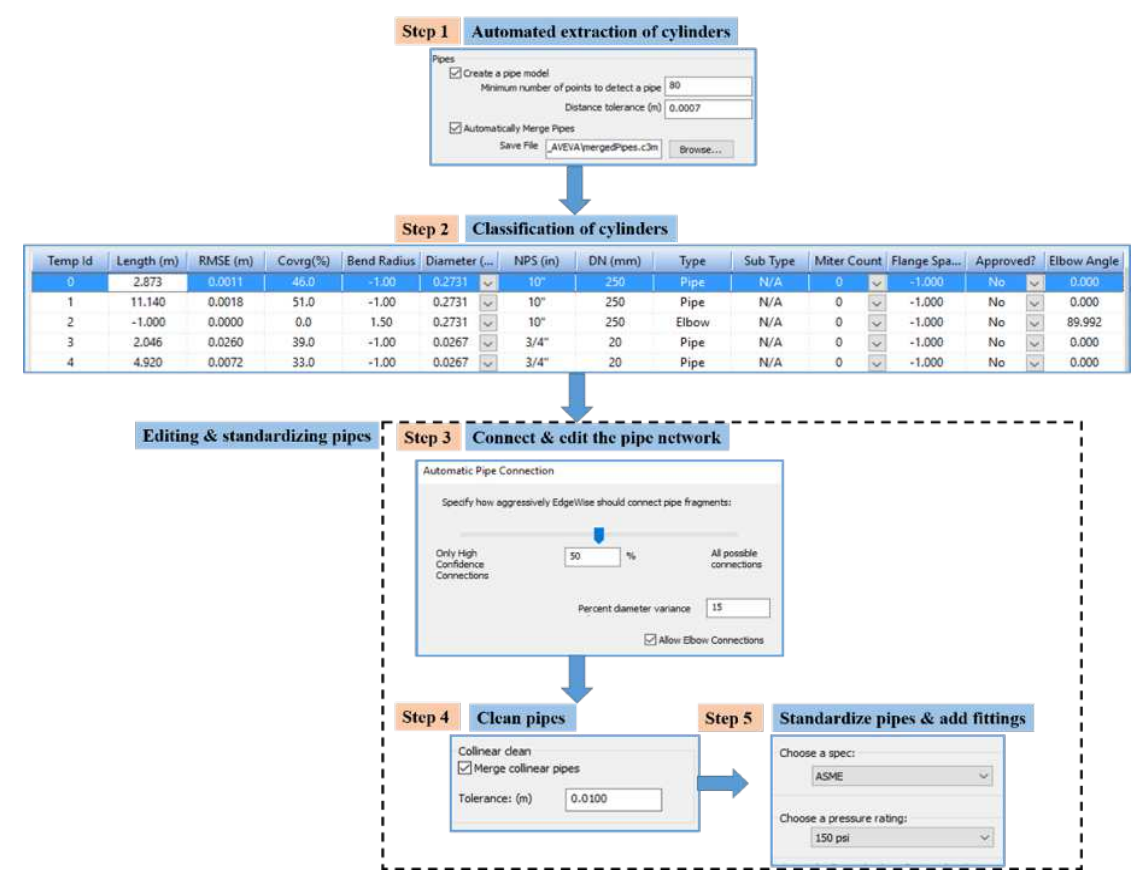

Fig. 2. Workflow of pipeline detection, classification and editing steps in EdgeWise

Table 3. Modelling time needed for each modelling task for each dataset and average time per object (min/object)

\begin{tabular}{|c|c|c|c|c|c|}
\hline & $\begin{array}{l}\text { Typical } \\
\text { facility } \\
\text { Room } 1\end{array}$ & $\begin{array}{l}\text { Typical } \\
\text { facility } \\
\text { Room } 2\end{array}$ & $\begin{array}{l}\text { Water } \\
\text { Facility }\end{array}$ & $\begin{array}{l}\text { Petro- } \\
\text { chemical } \\
\text { plant }\end{array}$ & \\
\hline Modelling Task & \multicolumn{4}{|c|}{ Time (min) } & $\begin{array}{c}\text { Average } \\
\text { time } \\
\text { (min) }\end{array}$ \\
\hline $\begin{array}{l}\text { Automated extraction } \\
\text { of cylinders }\end{array}$ & $1.5^{*} 10^{-3}$ & $1.5 * 10^{-3}$ & $7.1 * 10^{-3}$ & $1.4 * 10^{-3}$ & $3.3 * 10^{-3}$ \\
\hline $\begin{array}{l}\text { Semantic classification } \\
\text { of cylinders } \mathbf{s}^{\text {b }}\end{array}$ & 0.20 & 0.47 & 0.17 & 0.12 & 0.24 \\
\hline $\begin{array}{l}\text { Manual extraction \& } \\
\text { editing of pipes }\end{array}$ & 0.69 & 2.37 & 2.43 & 1.22 & 1.68 \\
\hline
\end{tabular}

${ }^{\mathrm{a}}$ per cylinder* point, ${ }^{\mathrm{b}}$ per cylinder, ${ }^{\mathrm{c}}$ per pipe 
The time for semantic classification was 0.24 minutes per cylinder on average. Manual extraction and editing of pipes was the most time-intensive step, since we needed 1.68 minutes per pipe on average to manually add missing pipes and edit the existing ones. The observations show that the manual effort to classify and extract pipes was 1.92 minutes per cylinder on average, which is the summation of two subsequent steps, semantic classification of cylinders and manual extraction and editing of pipes. This is almost three times the time needed for automated extraction of cylinders by the software.

A variation of the time needed for automated extraction of cylinders between the water facility and the other datasets is observed. This discrepancy is attributed to the fact that the water facility is an outdoor facility, requiring the most processing time compared to the other datasets. Technically, outdoor scenes are inherently more occluded and incomplete exhibiting extreme variations in point density [19]. These effects are mitigated by the limited size and constrained shape of rooms. The two rooms of the typical industrial facility were processed at the same time in our operating system, for this reason the time required for automated extraction is the same as shown in Table 3. Manual modelling of the second room of this facility required the most modelling time compared to the first room. This is due to cluttered pipelines, which resulted in the largest Room Mean Square Error (RMSE) of the cylinder diameters, as shown in Table 4. This clutter is attributed to the reflective surface of pipelines. Manual extraction and editing of pipes in the water facility is another modelling time outlier. Highly occluded pipelines are the primary reason for this outlier, since they have the lowest average coverage (26.5\%), compared to the other projects. The diameter of pipelines in the water facility was significantly larger, since most pipes are used for sewage purposes. These observations show that manually detected pipes have larger average diameter $(0.617 \mathrm{~m})$ compared to automatically extracted cylinders $(0.315 \mathrm{~m})$ for the same dataset. This means that it is difficult for the software to identify cylinders with large diameters.

Table 4. Root Mean Square Error (RMSE) of the radius and coverage (\%) of automatically detected cylinders in each dataset and average values

\begin{tabular}{lcc}
\hline $\begin{array}{c}\text { Automatically detected } \\
\text { cylinders in: }\end{array}$ & RMSE of the cylinder radius (m) & Coverage (\%) \\
\hline Typical facility - Room 1 & $1.7 * 10^{-3}$ & 32.5 \\
Typical facility - Room 2 & $6.7 * 10^{-3}$ & 30.2 \\
Water facility & $1.9 * 10^{-3}$ & 26.5 \\
Petrochemical plant & $4.2 * 10^{-3}$ & 27.6 \\
\hline Average & $3.6 * 10^{-3}$ & 29.2 \\
\hline
\end{tabular}

RMSE and coverage percentages for each extracted cylinder are calculated in the "SmartSheet", provided in EdgeWise. Table 4 summarizes their average values for all case studies. The results show that the first room of the typical industrial facility 
10

has the lowest RMSE, meaning that the extracted cylinders fit well the corresponding points of the cylinders. The average coverage area of cylinders in all case studies is around a quarter of the cylinder $(29.20 \%)$, which is the reason that many cylinders are not automatically extracted.

The performance of the software is evaluated based on the two-following metrics, precision and recall [20],

$$
\begin{gathered}
\text { Precision }=\mathrm{TP} /(\mathrm{TP}+\mathrm{FP}) \\
\text { Recall }=\mathrm{TP} /(\mathrm{TP}+\mathrm{FN})
\end{gathered}
$$

Where TP are the number of objects that are automatically detected as pipes and were correctly inspected as pipes, FP are the number of objects that are detected as pipes, but we classified them as other cylindrical objects (for instance handrails, circular hollow steel sections to name a few) and FN are the number of objects that are pipes but were not automatically detected as pipes. Those pipes were manually extracted and added to the model. 5.

The performance metrics obtained from our four sample datasets are given in Table

Table 5. Average performance metrics of pipe and cylinder detection

\begin{tabular}{lcccc}
\hline & Pipe detection metrics & \multicolumn{2}{c}{ Cylinder detection metrics } \\
\hline Dataset & Recall (\%) & $\begin{array}{c}\text { Precision } \\
\mathbf{( \% )}\end{array}$ & Recall (\%) & $\begin{array}{c}\text { Precision } \\
\mathbf{( \% )}\end{array}$ \\
\hline Typical facility Room 1 & 80.1 & 27.9 & 69.3 & 48.2 \\
Typical facility Room 2 & 59.5 & 54.6 & 100.0 & 22.0 \\
Water facility & 33.3 & 36.4 & 87.3 & 86.4 \\
Petrochemical plant & 59.6 & 69.3 & 45.7 & 91.9 \\
\hline \multicolumn{1}{c}{ Average } & 58.1 & 47.0 & 75.6 & 62.1 \\
\hline
\end{tabular}

According to precision, out of all the automatically detected cylinders only an average of $47 \%$ in all case studies correspond to pipes, whereas the rest were other cylindrical objects. The average recall was $58.1 \%$, meaning that only $58.1 \%$ of all pipes existing in a typical facility will be automatically detected. The results show that the water treatment facility, which is an outdoors facility, has the lowest recall, being $33.3 \%$. The low performance metrics of this dataset, compared to the other 
ones, can be attributed to increased noise. The low precision of pipes in the first room of the typical facility $(27.9 \%)$ is attributed to a larger number of FPs (roof tiles), which were wrongly detected as pipes.

The same metrics were measured for cylinders. The only difference in the metrics used is that precision is defined as the number of automatically detected cylinders out of all the detected cylinders, whereas recall is the number of automatically detected cylinders out of all other automatically detected non-cylindrical shapes. The recall of cylinders is high for all datasets except the petrochemical plant (45.7\%), which is attributed to low scan completeness of this dataset and increased clutter. The average recall for the four datasets is $75.6 \%$ indicating the advantage of the software to extract this primitive shape. The precision of cylinders is also $15 \%$ higher compared to that of pipes, since the software is designed to detect cylindrical shapes. The lowest precision $(22 \%)$ is observed for the second room of the typical industrial facility, which is attributed to corrugated shapes in the roof that were incorrectly modelled as cylinders. The same trend (low precision of about $48 \%$ ) is observed for the first room of the facility for the same reason.

Representative 3D models obtained from the room of the petrochemical plant, two rooms of a typical industrial facility and the water treatment plant are presented in Fig. 3, Fig. 4, Fig. 5 and Fig. 6. The initial point cloud, the automated pipeline extraction output and the final 3D model that was obtained after manual modelling of the most frequent pipeline elements, structural sections and electrical conduit are presented in the same Figures. These 3D models are not the complete 3D models of the facilities, but the subsets used for the evaluation purposes of this paper.

\subsection{EdgeWise evaluation for modelling of structural components}

Software packages used for extraction of structural elements have been developed by ClearEdge3D (2017). EdgeWise Structural is used for our evaluation in this work. The most frequent structural elements that were identified in our previous work [11] (circular hollow sections, channels, solid bars, I-beams) are modelled in the four case studies.

The user selects the I-beam, Channel and RoundTubing tools to manually extract the respective elements. The user can also create custom standards for shapes that do not exist on the standards list. The "Pattern Extract" tools extract groups on repeatable elements of the same object type. The extracted sections are then inspected for accuracy in the "SmartSheet". The workflow of the manually modelled structural sections is summarized in Fig. 7.

The standards that were used for this evaluation were taken from the American Institue of Steel Construction (AISC) manuals. The authors also used the "Autofit" tool to extract the correct size of the specified section automatically. Precision and recall metrics were not used herein, since the procedure is manual. 

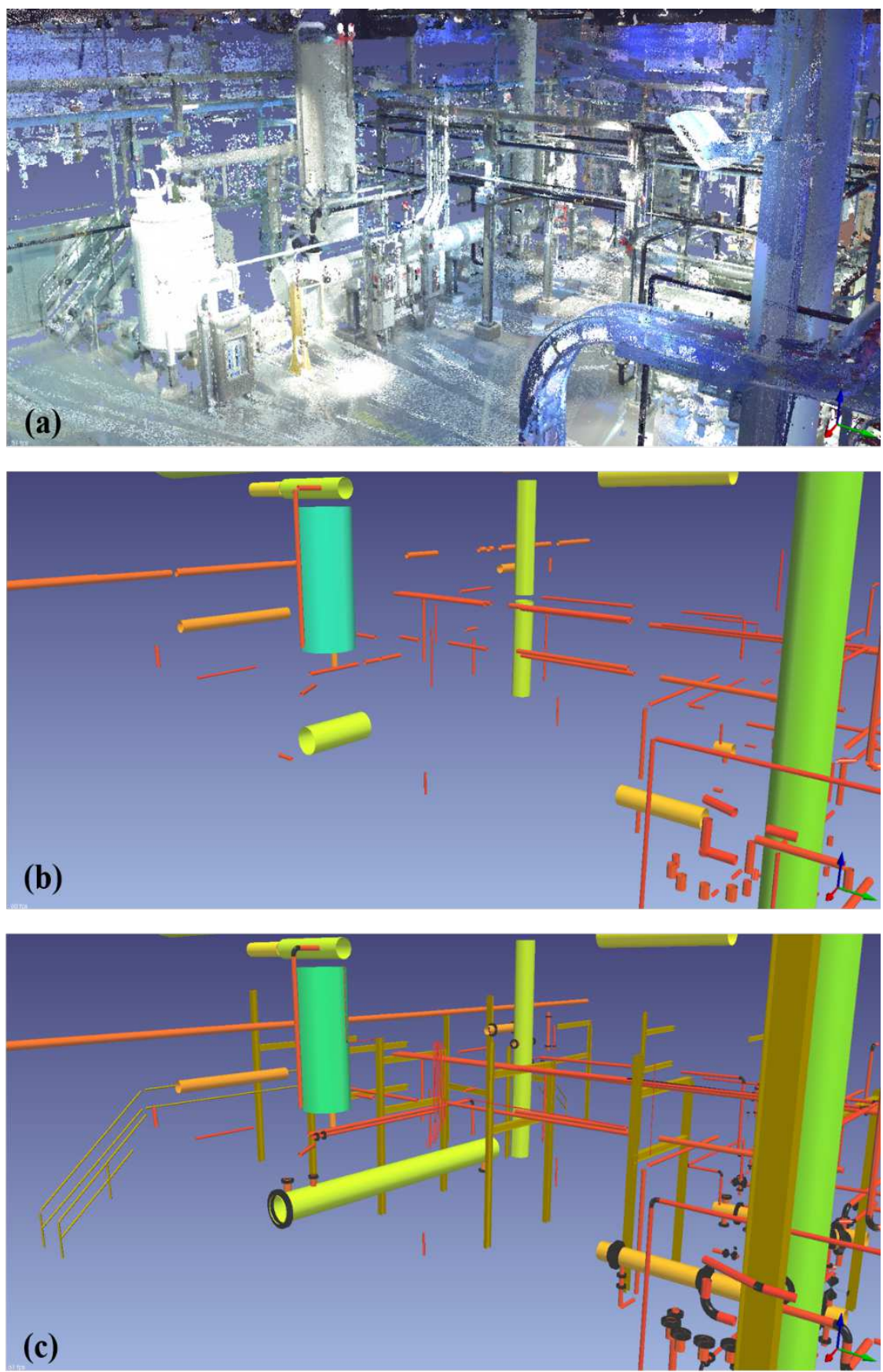

Fig. 3. Input point cloud, (b) automated cylinder extraction in EdgeWise Plant/MEP and (c) 3D model after manual modelling of pipes, structural elements and electrical conduit for a room of a petrochemical plant (dataset provided by AVEVA Group Plc.). 

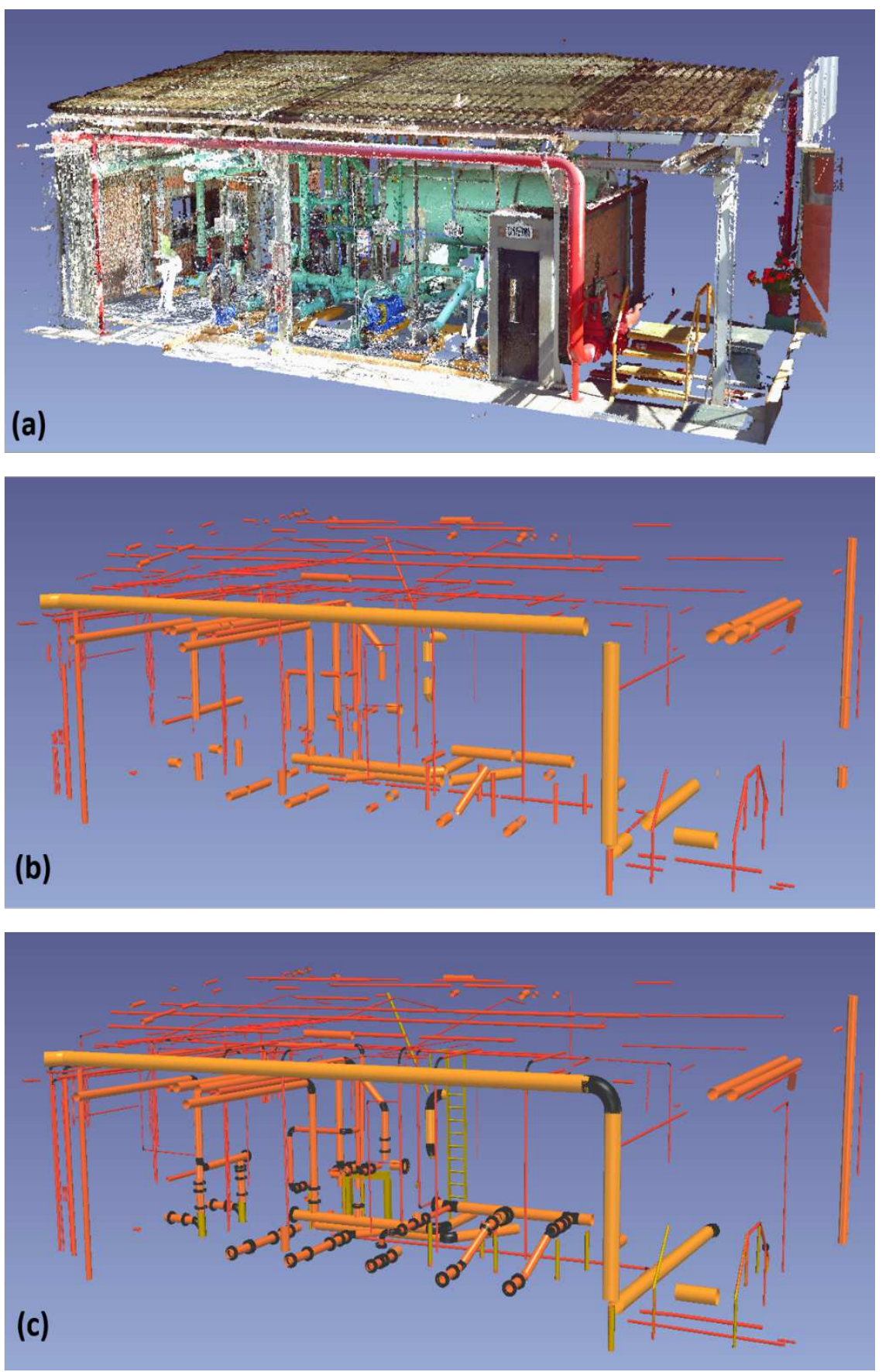

Fig. 4. Input point cloud, (b) automated cylinder extraction in EdgeWise Plant/MEP and (c) 3D model after pipe, structural and electrical conduit extraction for the first room of an industrial facility (dataset provided by AVEVA Group Plc.) 

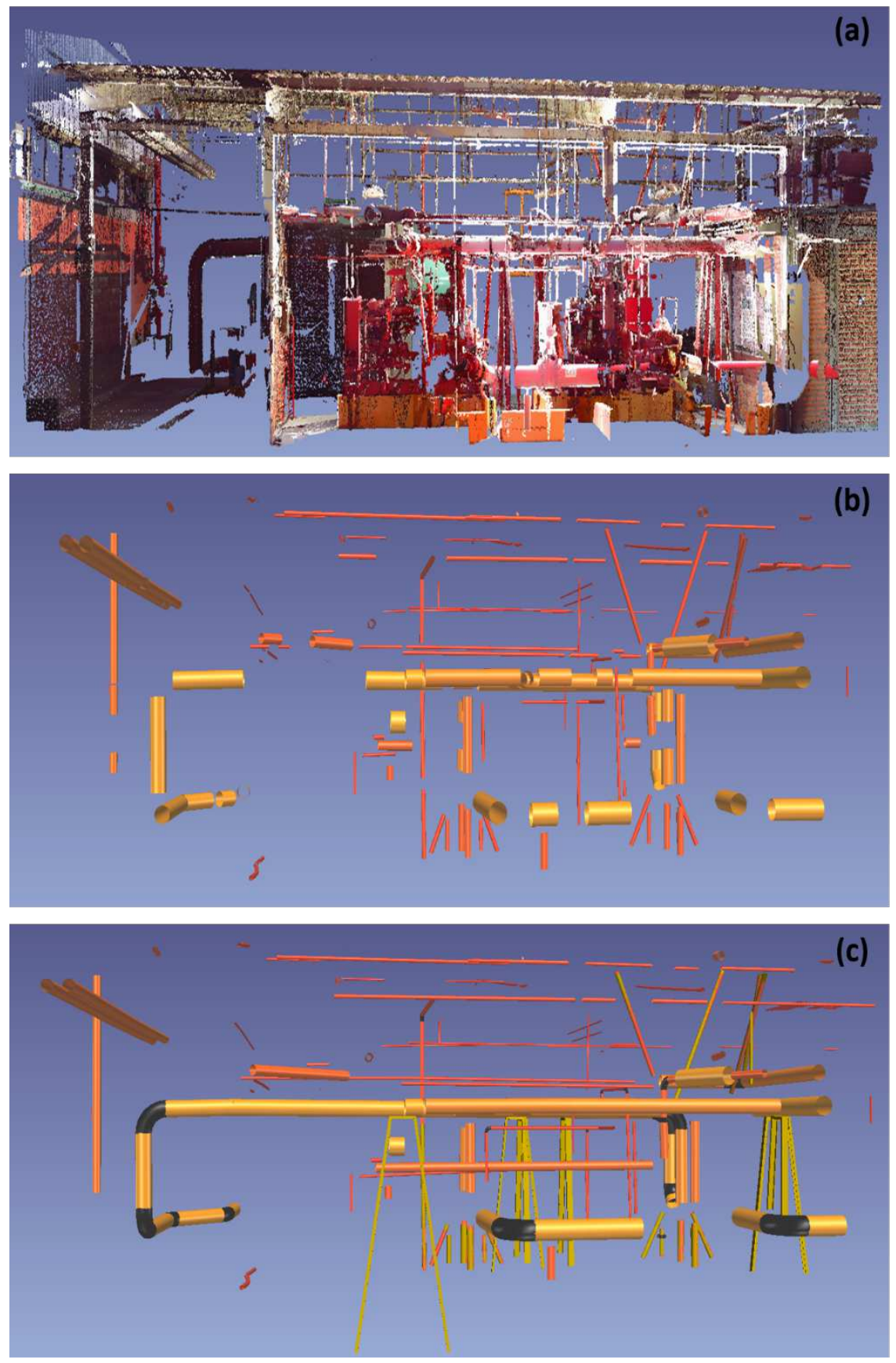

Fig. 5. Input point cloud, (b) automated cylinder extraction in EdgeWise Plant/MEP and (c) 3D model after pipe, structural and electrical conduit extraction for the second room of an industrial facility (dataset provided by AVEVA Group Plc.) 

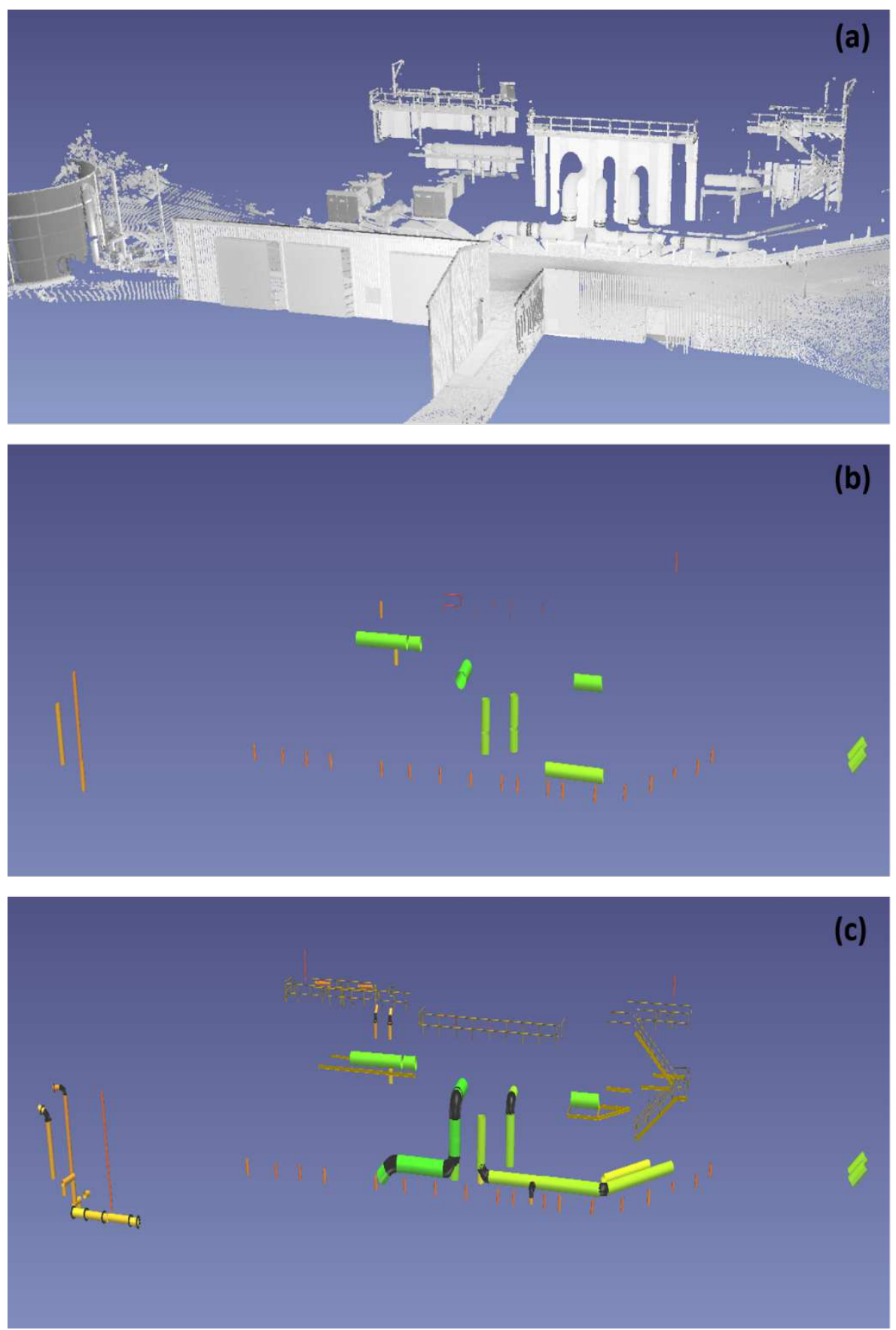

Fig. 6. Input point cloud, (b) automated cylinder extraction in EdgeWise Point/MEP and (c) 3D model after pipe, structural and electrical conduit extraction for a water treatment facility in Cambridge (dataset acquired by the authors) 
16

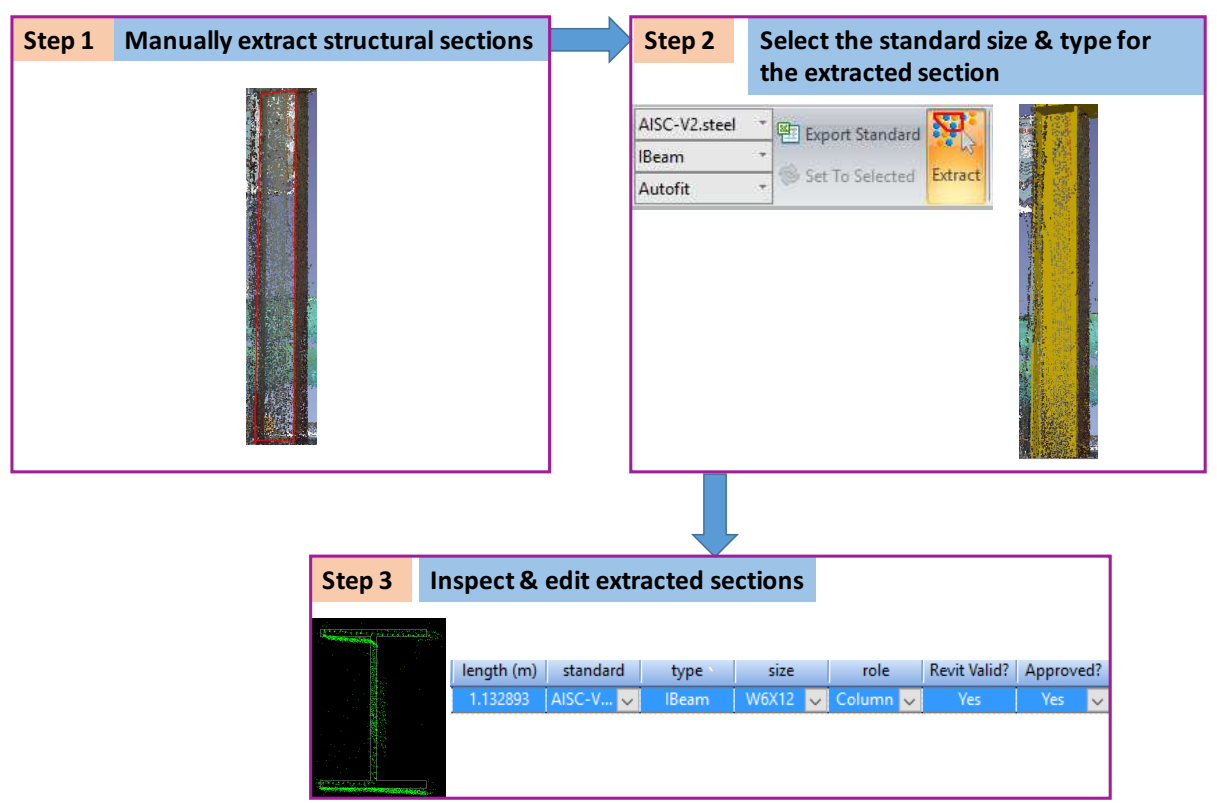

Fig. 7. Workflow of the manual modelling of structural elements in EdgeWise Structural.

\subsection{Overall performance of state-of-the-art modelling software}

The performance of state-of-the-art modelling software is summarized in Table 6 . This Table shows that fitting of the most frequent object types has been solved by commercial software like EdgeWise, since known geometric shapes are automatically fitted to the selected point clusters. Automated primitive shape detection of cylinders has partially been solved since the results showed $75 \%$ recall and $62 \%$ precision in EdgeWise. Non-cylindrical shapes are manually extracted, and classification of all object types has not been achieved.

Table 6. Performance of state-of-the-art software packages on each modelling step for the most frequent object types

\begin{tabular}{lccc}
\hline $\begin{array}{c}\text { Industrial object } \\
\text { type }\end{array}$ & $\begin{array}{c}\text { Primitive shape } \\
\text { extraction }\end{array}$ & $\begin{array}{c}\text { Semantic labelling } \\
\text { (classification) }\end{array}$ & Fitting \\
\hline Straight pipe & Partially solved & Not solved & Solved \\
CHS $^{\text {a }}$ & Partially solved & Not solved & Solved \\
Channel & Not solved & Not solved & Solved \\
Conduit & Partially solved & Not solved & Solved \\
I-beam & Not solved & Not solved & Solved \\
Valve & Not solved & Not solved & Solved \\
Elbow & Not solved & Not solved & Solved \\
Flange & Not solved & Not solved & Solved \\
Angle & Not solved & Not solved & Solved \\
\hline
\end{tabular}

${ }^{\mathrm{a}}$ Circular Hollow Section (CHS) 
The 3D models can be exported to Revit, in order to obtain IFC models for interoperability purposes between different software packages. However, we observed that reducers, valves, flanges, angles and some channels (C3 and C4 according to the American Institute of Steel Construction standards - AISC) cannot be exported in Revit. Models containing straight elements with length less than $4 \mathrm{~mm}$ cannot also be transferred to Revit.

Pipes, conduit and Circular Hollow Sections (CHSs) were also modelled manually in Revit to compare the man-hours needed for their shape extraction through this manual process. 30 objects were modelled in each category and their average modelling times were measured. The workflow of manual modelling in a software such as Revit entails three steps: (a) manual segmentation of the desired object in a point cloud visualization software such as CloudCompare, (b) export of the points in Autodesk Recap to obtain the appropriate format and then (c) modelling in Revit. Revit 2017 was used for this evaluation. The parameters of the cylinders (radius and length) are chosen based on the modeler's discretion. Non-cylindrical objecs were not modelled in Revit since their extraction in EdgeWise is manual, thus a comparison with Revit is redundant.

\subsection{Results}

The time needed to model the above-mentioned object types is measured in the same operating system as stated above for pipeline, structural and electrical object types. The average modelling time per object for the most frequent object types is calculated. The manual modelling time of cylindrical objects is broken down to the two steps investigated above; shape extraction and semantic classification. Knowing the average number of objects of a specific type in a typical facility, we calculate the average modelling time for each object type and each modelling step where applicable. Fig. 8 shows the modelling time/object in minutes and Fig. 9 the estimated total man-hours for modelling of the same object types in a typical industrial facility in hours.

Fig. 8 shows that manual extraction of straight pipes in EdgeWise is the most timeintensive task compared to semantic classification for pipes and requires 1.68 $\mathrm{min} / \mathrm{straight}$ pipe. Manual extraction of channels is also a laborious task compared to the manual extraction of all other object types, requiring $1.78 \mathrm{~min} / \mathrm{channel}$ due to the complexity of their shape. Although some of the CHSs are automatically extracted, it is difficult to identify them manually, since they are usually pipe supports and handrails, which are significantly occluded. For instance, pipe supports are occluded due to pipelines that run on top of them. This is the reason for intensive modelling time $(0.93$ $\mathrm{min} / \mathrm{CHS}$ ). Semantic classification of cylinders is not a time-intensive step, requiring less than $0.5 \mathrm{~min} /$ cylinder on average.

Given the average frequencies of objects in each type obtained in [11] for a sample facility of 100,000 objects, Fig. 9 shows that pipes require the most modelling time on average (around 800 hours) for this sample facility of 25,183 pipes. It is important to note that, although automated extraction of cylinders has been partially achieved by EdgeWise Plant/MEP, modelling of pipelines takes still substantial amount of time. The cylindrical shape is the most frequent geometric shape, thus the modelers' effort 
18

to distinguish electrical conduit, CHSs, handrails and other cylindrical objects from straight pipes is significant.

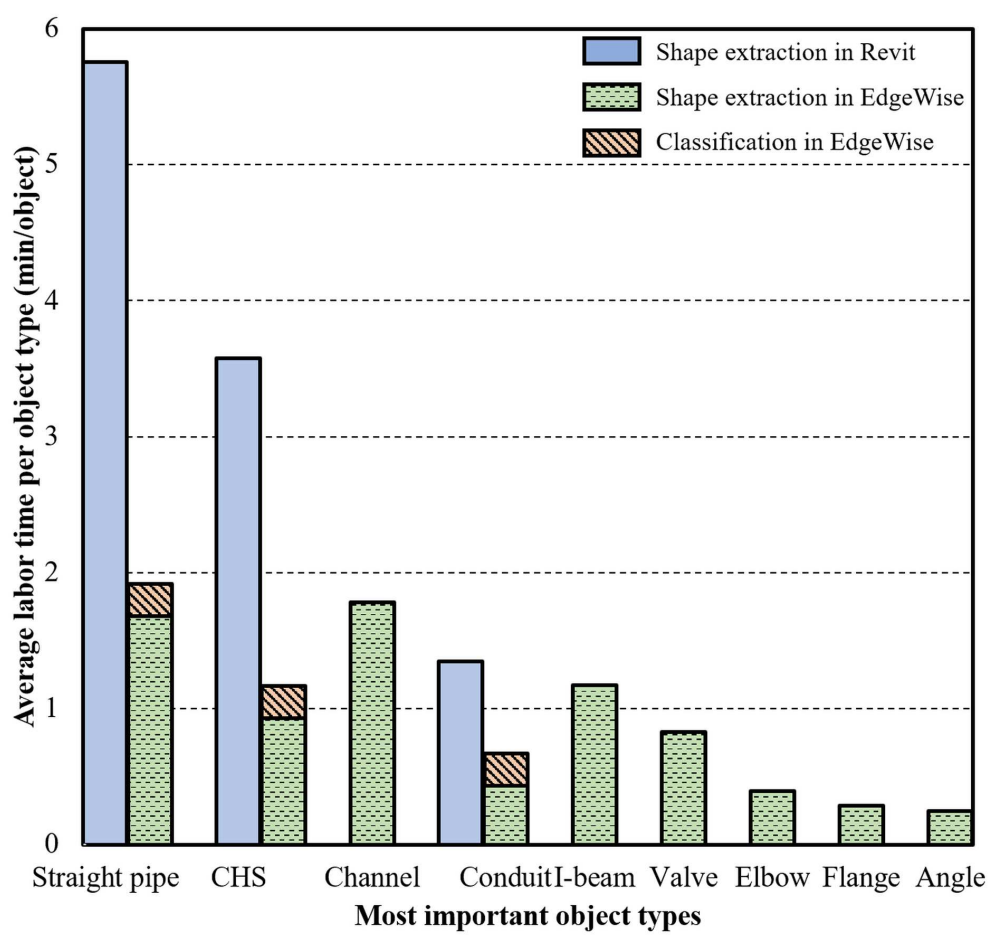

Fig. 8. Average modelling labor time per object (min/object) for the most frequent object types

Although electrical conduit is the most prevalent object type in industrial plants (24.3\% in a typical plant, [11]), it takes less man-hours to model it compared to a straight pipe. This is attributed to the design of electrical conduit that places many cylinders closely to each other. This makes it easier for the modeler to identify them, thus the modelling time is reduced.

Flanges and elbows do not require substantial time $(0.28$ and $0.39 \mathrm{~min} / \mathrm{object}$ respectively) as shown in Fig. 8, although the user manually adds them in the pipeline model. We observe that once the piping network is identified, the addition of fittings is a quick task that does not necessarily need to be automatically modelled. Angles require the least amount of time, being less than $0.25 \mathrm{~min} /$ angle, which is attributed to their simple geometry compared to I-beams or channels.

The total labor hours for manual modelling of an example industrial facility with 100,000 objects of the above categories are estimated to be 12 person-months. This finding is based on the following assumptions: (a) one trained modeler for all case studies, (b) the working hours are assumed to be 8 hrs/day, 5 days/week and (c) the operating system is as specified above. The same metric for cylinder extraction and classification is 8.5 person-months using EdgeWise as explained above. The confi- 
dence intervals for the average manual modelling time of pipes, conduit and CHSs are calculated since the selection of parameters depends on the modeler's discretion. Pipes were manually modelled in Revit in $5.8 \pm 1$ minutes with $99 \%$ confidence level. Conduit and CHSs were modelled in $1.3 \pm 0.75$ and $3.6 \pm 0.4$ minutes respectively. This means that the modelling time does not change substantially for any of these object categories.

The modeller was trained to model MEP and structural objects before starting the modelling task. 100 instances of each object type were modelled as a training exercise before the modeller started to perform this task. The theoretical example of 100,000 objects was chosen, given that the average total number of these object types is 191,991 as obtained from the case studies investigated in [11].

We observe that $64 \%$ of the man-hours needed for manual modelling of cylinders are saved by using the state-of-the-art software, EdgeWise, compared to conventional manual modelling platforms such as Revit. The results also show that $67 \%$ of manual modelling time is saved for pipe modelling in EdgeWise. The case study of 100,000 objects shows that 2,400 labor hours are saved when modelling cylinders in EdgeWise. This is crucial especially for these facilities, since the time required to take decisions for maintenance and refurbishment is limited due to continuous production flow.

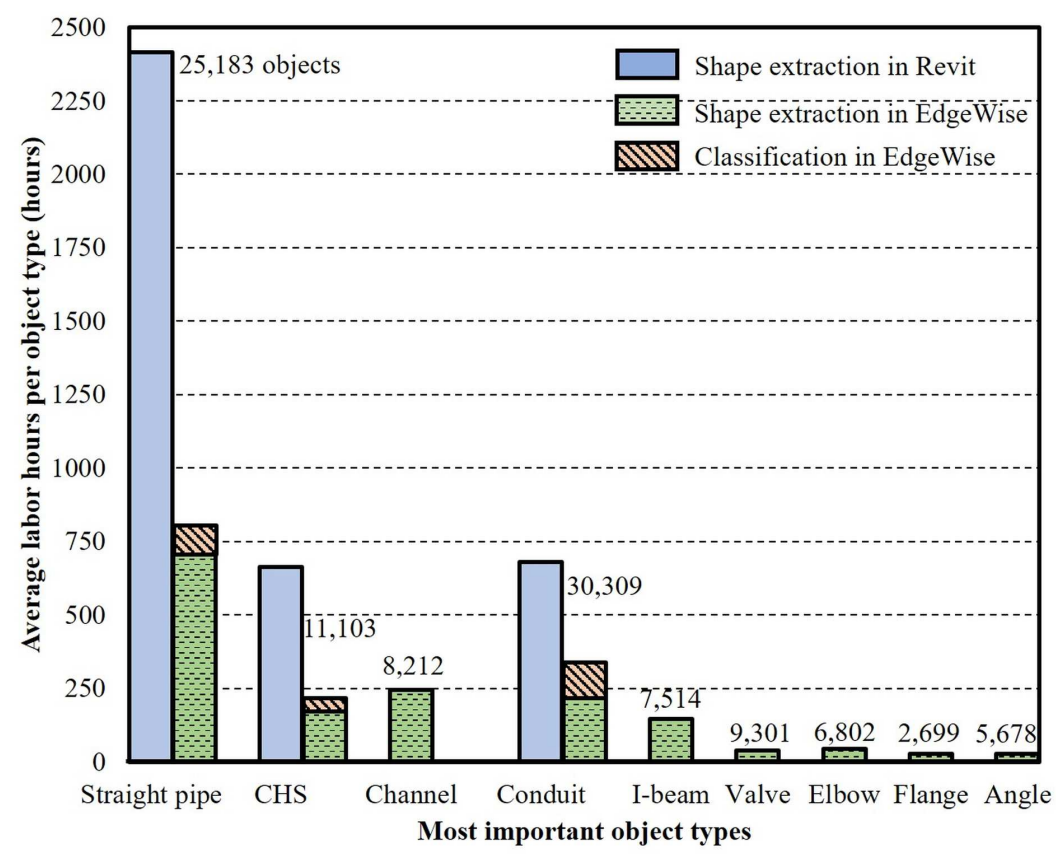

Fig. 9. Average modelling labor hours per object type for the most frequent objects of an example facility with shown numbers of objects 


\section{Conclusions}

The modelling time and shape extraction of the ten most frequent industrial object types are evaluated in the semi-automated, state-of-the-art software, EdgeWise. The results showed that cylindrical objects (straight pipes, electrical conduit and circular hollow sections) require $80 \%$ of the Total Modelling Hours (TMH) of the ten most frequent object types in EdgeWise.

The results of this paper show that current practice has achieved primitive shape extraction for straight pipes, elbows and conduit semi-automatically. However, semantic labelling of each object type is not performed in the state-of-the-art modelling packages. EdgeWise has substantially facilitated 3D modelling of industrial plants according to the findings discussed above. However, it has some limitations, which can be summarized as follows:

1. The modeler should identify the structural elements manually or define the location of an object roughly in the point cloud to fit it.

2. Detection of cylinders has only been partially solved, since cylinders are detected with $75 \%$ recall and $62 \%$ precision. The same metrics for pipes are $58 \%$ and 47 $\%$ respectively.

3. EdgeWise and all other 3D modelling software platforms do not enrich the 3D geometric primitives with semantic labels and topological relationships. Engineers are required to manually implement the semantic labels of the components of the 3D model.

4. Data inconsistency between different software platforms impedes modelers from exchanging data between different AI-BIM platforms. These software packages are not designed to provide a final output in an open and generic schema.

The contribution of this paper is the measurement of the performance of state-ofthe-art software and more specifically EdgeWise. This uncovered (a) the substantial performance of this software in detecting cylinders, (b) the inability of this software to (i) further classify cylinders into electrical conduit, pipes or CHSs and (ii) detect and further classify I-beams, channels, elbows, flanges, valves and angles in spite of their high frequency in an industrial facility.

Direct implications of modelling the most frequent industrial objects of [11] are assessed based on modelling time. The results of the evaluation of EdgeWise showed that semi-automatically modelling cylinders will reduce man-hours needed for modelling those by $64 \%$. This can have a direct impact for industrial facility managers, since every hour of as-is modelling time is crucial for the operation of the plant in unprecedented circumstances (failures of critical objects, retrofitting operations and plant expansion).

Indirect implications of prioritizing object types are reductions of the modelling cost, since man-hours of modelers will be reduced. Although there is no way to calculate the exact cost of overestimated severity of industrial inspections and maintenance, it is reasonable to predict that maintenance of industrial plants will be substantially facilitated once AI-BIMs are easy to develop and the costs do not counteract the benefits of their creation. Poor maintenance of these assets does not always affect the 
asset's territory but also impacts nearby regions and puts lives of the public living close by at serious risk.

The presented research has room for improvement and some limitations of this study can direct future research. This study focused on the industrial objects that are important to model, however methods on how to automatically model those were not investigated. Current research efforts were compared with commercial tools like EdgeWise showing no significant advances in terms of automated detection of cylinders. Future work involves implementation of automated machine learning algorithms for all the most frequent object types to minimize the modelling time. Application of these algorithms for hundreds of classes of different objects is a difficult multiclassification problem, that will be substantially benefited from the results of this exploratory research for the most frequent objects to model in these complex environments. Overall, a training library of the object classes that are critical for industrial facility operations, frequent in industrial environments and laborious to model can be established to assist further research aimed at automated detection of these classes. Application of the findings of this paper will guide researchers on investigating methods for automatically modelling these objects.

\section{Acknowledgements}

We thank our colleague Graham Miatt, Senior Software Engineer in LFM Software from AVEVA Group Plc., who provided insight and expertise that greatly assisted this research. We also express our gratitude to Otto Weiberth, Managing Director of ClearEdge3D, Inc., who provided EdgeWise Suite for evaluation. The research leading to these results has received funding from the Engineering and Physical Sciences Research Council (EPSRC). AVEVA Group Plc. and BP International Centre for Business and Technology (ICBT) partially sponsor this research under grant agreements RG83104 and RG90532 respectively. We gratefully acknowledge the collaboration of all academic and industrial project-partners. Any opinions, findings, and conclusions or recommendations expressed in this material are those of the authors and do not necessarily reflect the views of the institutes mentioned above.

\section{References}

1. Volk R, Stengel J, Schultmann F (2014) Building Information Modeling (BIM) for existing buildings - Literature review and future needs. Autom. Constr. 38:109-127

2. Tornincasa S, Monaco F Di (2010) The Future and the Evolution of CAD. Int Res Conf 'Trends Dev Mach Assoc Technol 18

3. Cabinet Office H (2011) Government Construction Strategy. Construction 96:43 . doi: Vol 19

4. Rabbani T, van den Heuvel F a, Vosselman G (2006) Segmentation of point clouds using smoothness constraint. Int Arch Photogramm Remote Sens Spat Inf Sci - Comm V Symp 'Image Eng Vis Metrol 36:248-253

5. Son H, Kim C, Kim C (2013) Knowledge-based approach for 3D reconstruction of asbuilt industrial plant models from laser-scan data. Int Symp Autom Robot Constr 
$756: 885-893$

6. Kawashima K, Kanai S, Date H (2014) As-built modeling of piping system from terrestrial laser-scanned point clouds using normal-based region growing. J Comput Des Eng 1:13-26 . doi: 10.7315/JCDE.2014.002

7. Veldhuis H, Vosselman G (1998) The 3D reconstruction of straight and curved pipes using digital line photogrammetry. ISPRS J Photogramm Remote Sens 53:6-16 . doi: 10.1016/S0924-2716(97)00031-2

8. $\quad$ Sanders FH (2001) 3D laser scanning helps Chevron revamp platform. 92-98

9. Fumarola M, Poelman R (2011) Generating virtual environments of real world facilities: Discussing four different approaches. In: Automation in Construction. pp 263-269

10. Hullo J-F, Thibault G, Boucheny C, et al (2015) Multi-Sensor As-Built Models of Complex Industrial Architectures. Remote Sens 7:16339-16362 . doi: $10.3390 /$ rs 71215827

11. Agapaki E, Brilakis I (2017) Prioritising object types of industrial facilities to reduce as-is modelling time. In: Proceeding of the 33rd Annual ARCOM Conference, 4-6 September 2017. Cambridge, U.K., pp 402-411

12. Douglas JM (1988) Conceptual design of chemical processes

13. FARO (2017) FARO. https://www.faro.com/en-in/products/construction-bimcim/faro-pointsense/features/

14. Ahmed MF, Haas CT, Haas R (2014) Automatic Detection of Cylindrical Objects in Built Facilities. J Comput Civ Eng 28:4014009 . doi: 10.1061/(ASCE)CP.19435487.0000329

15. Patil AK, Holi P, Lee SK, Chai YH (2017) An adaptive approach for the reconstruction and modeling of as-built 3D pipelines from point clouds. Autom Constr 75:65-78 . doi: 10.1016/j.autcon.2016.12.002

16. Son H, Kim C, Kim C (2013) Knowledge-based approach for 3D reconstruction of asbuilt industrial plant models from laser-scan data. ISARC 756:885-893

17. Perez-Gallardo Y, Cuadrado JLL, Crespo ÁG, de Jesús CG (2017) GEODIM: A Semantic model-based system for 3D recognition of industrial scenes. In: Intelligent Systems Reference Library. pp 137-159

18. ClearEdge (2017) ClearEdge. http://www.screencast.com/users/ClearEdge3D

19. Hackel T, Wegner JD, Schindler K (2016) Contour Detection in Unstructured 3D Point Clouds. In: 2016 IEEE Conference on Computer Vision and Pattern Recognition (CVPR). pp 1610-1618

20. Powers DMW (2011) Evaluation: From Precision, Recall and F-Measure To Roc, Informedness, Markedness \& Correlation. J Mach Learn Technol 2:37-63 . doi: 10.1.1.214.9232 